\title{
The inflammasome protects?
}

Inflammatory bowel disease (IBD) increases the risk of developing colorectal cancer (CRC) and is a good example of the link between inflammation and cancer in the gastrointestinal tract. Several molecular players, including the Toll-like family of pattern recognition receptors (PRRs), have emerged as mediators of IBD and CRC. Reporting in The Journal of Experimental Medicine, Jenny Ting and colleagues now show that another

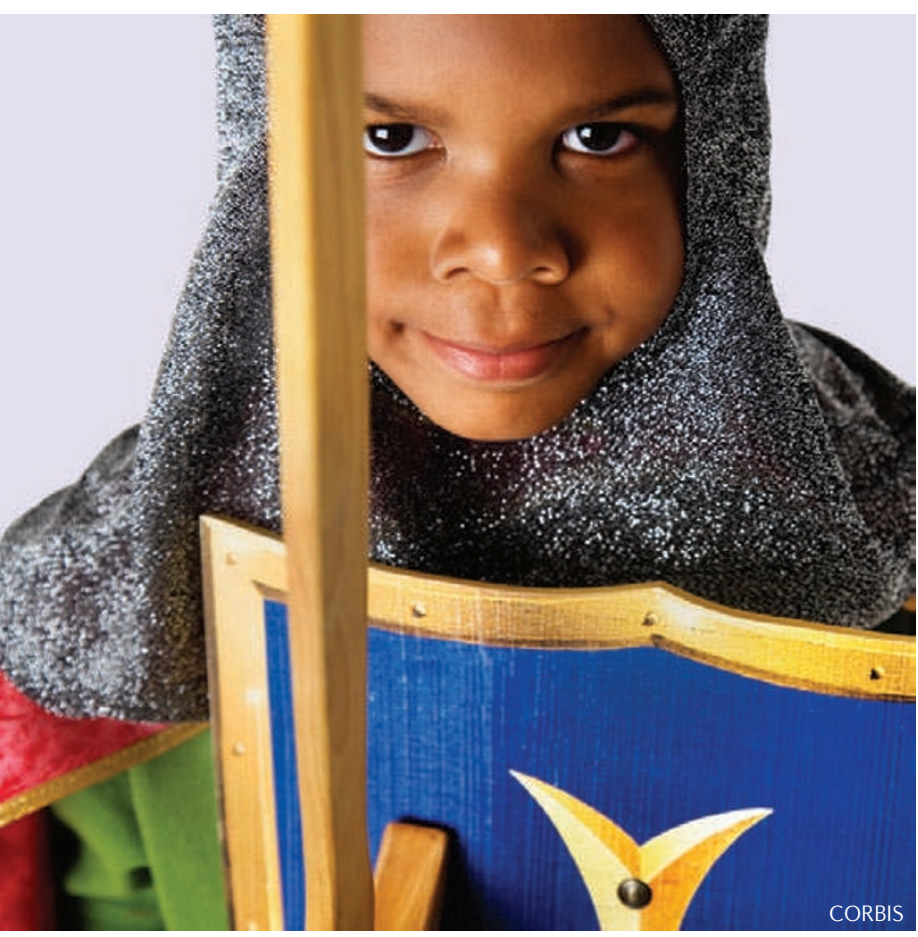

family of PRRs, the nucleotidebinding domain, leucine-rich repeatcontaining (NLR) proteins, function in both ulcerative colitis (UC) - one of the two main forms of IBD - and colitis-associated cancer (CAC).

NLRs regulate the maturation and secretion of the proinflammatory cytokines interleukin- $1 \beta$ (IL-1 $\beta$ ) and IL-18 through the formation of a caspase 1-activating inflammasome complex. This complex includes the adaptor protein PYCARD, which on NLR binding recruits procaspase 1 and facilitates its autocleavage to active caspase 1 . To determine the effects of the NLR inflammasome on both colitis and CAC, the authors made use of a mouse model that uses dextran sulfate sodium (DSS) to induce UC and azoxymethane (AOM) together with DSS to induce a disease that mimics CAC. Unexpectedly, when compared with wild-type mice, ${\text { Casp } 1^{-/-} \text {or Pycard }}^{-1-}$ mice had increased colon polyp formation, as well as increases in distal colon hyperplasia and dysplasia in the CAC model. Moreover, knock out of Casp1 or Pycard increased disease severity in models of acute and recurring colitis.

Given that two key components of the inflammasome protect against gastrointestinal tumorigenesis, which NLR is responsible for driving disease suppression? As common variants of NLRP3 are connected with an increased risk of developing the IBD Crohn's disease, this NLR was the first to be assessed in the CAC model. Like the Casp1 $1^{-/-}$and Pycard ${ }^{-/-}$mice, Nlrp3 $3^{-/-}$mice had increased tumour burden and a similar colon histopathology, but to a lesser extent, suggesting that other NLRs might also contribute to tumour suppression. However, knock down of Nlrc4 had no effect on either inflammation or gastrointestinal cancer. To uncover whether NLRP3 was affecting colonic epithelial cells or immune cells, bone marrow transplants were carried out. Expression of NLRP3 in haematopoietic cells, but not intestinal epithelial cells or stromal cells, was found to negatively regulate tumour formation.

At first glance, the protective role of the NLRP3 inflammasome is surprising, as one might expect that the proinflammatory cytokines produced would create favourable conditions for tumorigenesis. Therefore, the mechanism of this response awaits further elucidation.

Kira Anthony, Editor, NCI-Nature Pathway Interaction Database

ORIGINAL RESEARCH PAPER Allen, I. C. et al.

The NLRP3 inflammasome functions as a

negative regulator of tumorigenesis during colitis-associated cancer. J. Exp. Med. 12 Apr 2010 (doi:10.1084/jem.20100050) 